\title{
Covered Stenting Is an Effective Option for Traumatic Carotid Pseudoaneurysm with Promising Long-Term Outcome
}

\author{
Kai Wang, ${ }^{1, *}$ Xiao-xin Peng, ${ }^{2, *}$ Ao-fei Liu, ${ }^{1, *}$ Ying-ying Zhang, ${ }^{1}$ Jin Lv, Li Xiang, ${ }^{1}$ Yun-e Liu, Wei-jian Jiang ${ }^{1}$ \\ New Era Stroke Care and Research Institute, The PLA Rocket Force Characteristic Medical Center, Beijing, China \\ Department of Radiology, ${ }^{2}$ Beijing Jishuitan Hospital, Beijing, China
}

Objective : Covered stenting is an optional strategy for traumatic carotid pseudoaneurysm, especially in malignant conditions of potential rupture, but the long-term outcomes are not clear. Our aim was to determine if covered stenting is an effective option for traumatic carotid pseudoaneurysm with promising long-term outcomes.

Methods : Self-expanding Viabahn and balloon-expandable Willis covered stents were separately implanted for extra- and intracranial traumatic carotid pseudoaneurysm. The covered stent was placed across the distal and proximal pseudoaneurysm leakage under roadmap guidance. Procedural success was defined as technical success (complete exclusion of the pseudoaneurysm and patency of the parent artery) without a primary end point (any stroke or death within 30 days after the procedure). Longterm outcomes were evaluated as ischemic stroke in the territory of the qualifying artery by clinical follow-up through outpatient or telephone consultation and as the exclusion of the pseudoaneurysm and patency of the parent artery by imaging follow-up through angiography.

Results : Five patients with traumatic carotid pseudoaneurysm who underwent covered stenting were enrolled. The procedural success rate was $100 \%$. No ischemic stroke in the territory of the qualifying artery was recorded in any of the five patients during a mean clinical follow-up of $44 \pm 16$ months. Complete exclusion of the pseudoaneurysm and patency of the parent artery were maintained in all five patients during a mean imaging follow-up of $39 \pm 16$ months.

Conclusion : Satisfactory procedural and long-term outcomes were obtained, suggesting that covered stenting is an effective option for traumatic carotid pseudoaneurysm.

Key Words : Cerebrovascular trauma $\cdot$ Carotid artery injuries $\cdot$ Stents $\cdot$ Treatment outcome.

\section{INTRODUCTION}

Traumatic carotid injury lesion is categorized into types I-V, including nonhemodynamically significant intimal injuries, potentially hemodynamically significant dissections and hematomas, pseudoaneurysms, occlusions, and avulsion, ac-

- Received : October 9, 2019 •Revised : December 31, 2019 •Accepted : February 14, 2020

- Address for reprints : Wei-jian Jiang

New Era Stroke Care and Research Institute, The PLA Rocket Force Characteristic Medical Center, Xinwai Street 16th, Beijing 100088, China

Tel : +86-10-66343998, Fax : +86-10-66343998, E-mail : cjr.jiangweijian@vip.163.com, ORCID : https://orcid.org/0000-0003-1156-5720

*There authors contributed equally to this work.

This is an Open Access article distributed under the terms of the Creative Commons Attribution Non-Commercial License (http://creativecommons.org/licenses/by-nc/4.0) which permits unrestricted non-commercial use, distribution, and reproduction in any medium, provided the original work is properly cited. 
cording to Biffl et al. ${ }^{4)}$. Traumatic carotid pseudoaneurysms (type III), which indicate a mechanism of paravascular encapsulated hematomas communicating directly with the lumen of the artery (Fig. 1), may accidentally occur after blunt/penetrating trauma or iatrogenic injury. Because of the special formation mechanism and in the absence of a complete vascular wall structure ${ }^{23)}$, traumatic carotid pseudoaneurysm is more likely to lead to complications, such as severe ischemic and hemorrhagic events, neck compression symptoms, and even life-threatening rupture emergency. The natural history of pseudoaneurysms is controversial, but the subgroup with giant and irregular shapes, short-term enlargement, or intracranial locations is often attributed to malignant conditions. Sufficient attention and timely disposal are usually necessary.

Anticoagulation therapy alone has been shown to produce a paradoxical effect, with a lack of improvement of pseudoaneurysm angiograms but an increase in wall hematoma ${ }^{25}$. The earliest surgical treatment of proximal ligation can serve as a deterministic deconstructive strategy for pseudoaneurysm, but occlusion intolerance in the patient and retrograde flow to the pseudoaneurysm make it far from ideal. As an improvement, bypass procedures have been described for providing extra-anatomic reconstruction of the carotid artery. However,

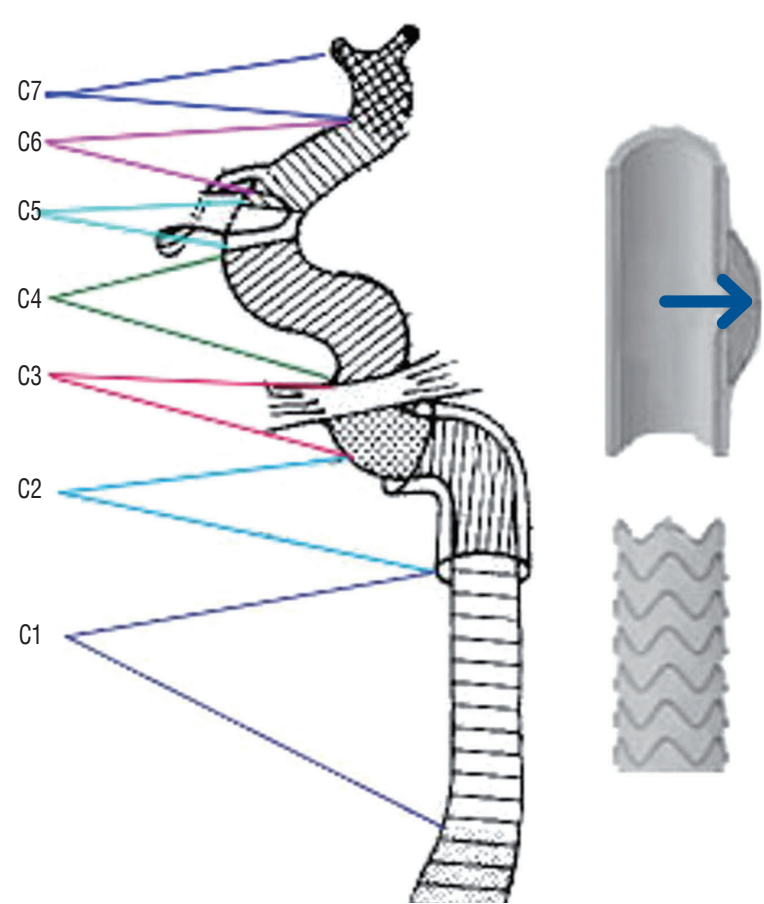

Fig. 1. Bouthillier segment classification of the carotid artery and a diagrammatic sketch of a pseudoaneurysm and covered stent. bypass procedures are not always easy to carry out because of exposure difficulties and the potential risk of stroke ${ }^{18)}$. Alternatively, the endovascular strategies of stenting or embolization became the frequent choice by interventional radiologists and have been reported to have promising short-term results $^{6,12)}$. However, for pseudoaneurysms with irregular shape and giant size, stenting or embolization usually show limited effect.

The new endovascular strategy of covered stenting has introduced a concept innovation for traumatic pseudoaneurysm that can tightly cover the leakage of the pseudoaneurysm and result in intra-aneurysmal thrombosis with preservation of the injured carotid artery (Fig. 1). Although several smallsized case series have reported promising immediate results, covered stenting of traumatic carotid pseudoaneurysm has not been widely used in most trauma centers, and little is known about the long-term outcomes ${ }^{15)}$. In the present study, we introduced the long-term follow-up results of covered stenting for traumatic carotid pseudoaneurysm, especially in malignant conditions, to further supplement the experience of craniocervical trauma management.

\section{MATERIALS AND METHODS}

\section{Patient inclusion}

The study was approved by the Institutional Ethics Committee (KY2013031). The subgroup of covered stenting for traumatic carotid aneurysms was retrieved from the institutional stenting database. The inclusion criteria were as follows : 1) patients with epistaxis, visual impairment, ptosis, or cervical mass after a history of blunt/penetrating trauma or iatrogenic injury and 2) carotid artery pseudoaneurysm involving the common carotid artery (CCA) or internal carotid artery (ICA) C1-C7, confirmed as criminal lesions by transcatheter cerebral angiography. Baseline data (gender, age, symptoms, etiology, location of lesions, size of pseudoaneurysms, and length of leakage) and follow-up results (clinical events, imaging findings and complications) were prospectively collected. All patients provided written informed consent.

\section{Indication and procedure of covered stenting}

Traumatic carotid pseudoaneurysms with giant and irregular shapes, short-term enlargement, or intracranial locations 
are considered malignant and are indicated for covered stenting. Interventional procedures were performed by the same experienced interventionist. Aspirin (100 mg/d) and clopidogrel $(75 \mathrm{mg} / \mathrm{d})$ were taken before the operation for 3 consecutive days, and all procedures were performed under general anesthesia. For CCA and extracranial segments of the ICA, the self-expanding Viabahn covered stent (W. L. Gore \& Associates Inc, Flagstaff, AZ, USA) was selected. To ensure good adherence with the parent artery, the stent diameter was selected mainly according to the larger diameter of the proximal and distal parent arteries. To avoid incomplete exclusion, the length of the stent must be greater than the width of the endoleak. A 6-French femoral sheath was changed to an 8-French catheter placed in the lesion side of the CCA. Under guidance of angiography road mapping, the covered stent was deployed across the distal and proximal ends of the pseudoaneurysm leakage. For intracranial ICA segment lesions, a balloon-expandable Willis covered stent (MicroPort Medical, Shanghai, China) was used. After positioning a 6-French guiding catheter in the ICA, a microguidewire was navigated distally. The Willis covered stent was then navigated and deployed across the corresponding lesion with the recommended pressure of $6 \mathrm{~atm}$. If residual endoleak was detected from immediate angiography, balloon reinflation was performed with 8 atm pressure. Digital subtraction angiography (DSA) and cranial computed tomography were performed for the evaluation of procedural success. The patient was monitored for signs and symptoms postprocedurally until discharge. If potential procedural-specific symptoms occurred, appropriate imaging was performed to ensure the absence of stroke, instent thrombosis, active bleeding or dissection. Heparin was given for 48 hours after the procedure. Patients were instructed to take aspirin and clopidogrel for 3 months, followed by aspirin alone indefinitely.

\section{Follow-up and outcome measures}

Clinical follow-up information was derived from daily patient exams until discharge, 30-day follow-up visits and subsequent clinic visits or telephone conversations until the end of December 2018. Follow-up angiography was scheduled after 6 months on a voluntary basis or when restenosis was clinically suspected. Procedural success was defined as technical success (complete exclusion of the pseudoaneurysm and patency of the parent artery) without primary endpoint (any stroke or death within 30 days after the procedure). Long-term outcomes were evaluated as ischemic stroke in the territory of the qualifying artery by clinical follow-up (outpatient or telephone consultation) and as recurrence of the pseudoaneurysm and patency of the parent artery by imaging follow-up (DSA/ computed tomography angiography [CTA]). Potential complications (including stroke, vessel injury, stent thrombosis, and in-stent stenosis) were documented in great detail.

\section{RESULTS}

The baseline data and outcome results are summarized in Table 1. From January 1, 2014 to December 1, 2017, a total of five patients ( $42 \pm 17$ years, three males) meeting the inclusion criteria were enrolled in the study. In etiology, two cases were direct craniofacial blunt trauma, and the other three cases were iatrogenic injury (underwent carotid endarterectomy or meningioma resection surgery). In the present series, pseudoaneurysm-related symptoms manifested as local pain, neck mass, headache, diplopia or asymptomatic. Through preoperative catheter angiography (Fig. 2), one case had a pseudoaneurysm of the CCA, one case of extracranial ICA, and the other three cases of the intracranial ICA. Pseudoaneurysm sizes ranged from $5.9 \times 3.3 \mathrm{~mm}$ to $21.7 \times 9.3 \mathrm{~mm}$.

For two extracranial pseudoaneurysms, self-expanding Viabahn covered stents $(6 \times 50 \mathrm{~mm}$ and $6 \times 50 \mathrm{~mm}$, respectively) were selected, while for three intracranial ICA pseudoaneurysms, balloon-expandable Willis covered stents $(4.5 \times 16 \mathrm{~mm}$, $3.5 \times 10 \mathrm{~mm}$, and $4.5 \times 13 \mathrm{~mm}$ ) were implanted (Table 1 ). No technical obstacle was encountered in the navigation and deployment of any of the stents. Immediately after stenting, complete exclusion of the pseudoaneurysm was achieved in four patients. In a 56-year-old case, endoleak into the pseudoaneurysmal cavity remained after the initial deployment of the Willis stent, but the endoleak disappeared after balloon reinflation (Fig. 2). No navigation-related adverse events occurred during the procedures. No stroke or death occurred within 30 days after the procedure. Procedural success was ultimately achieved in all patients (100\%).

During an average of $44 \pm 16$ months of clinical follow-up, no ischemic strokes in the territory of the qualifying artery were recorded in any of the cases (Table 1). During an average of $39 \pm 16$ months of imaging follow-up (DSA images available 


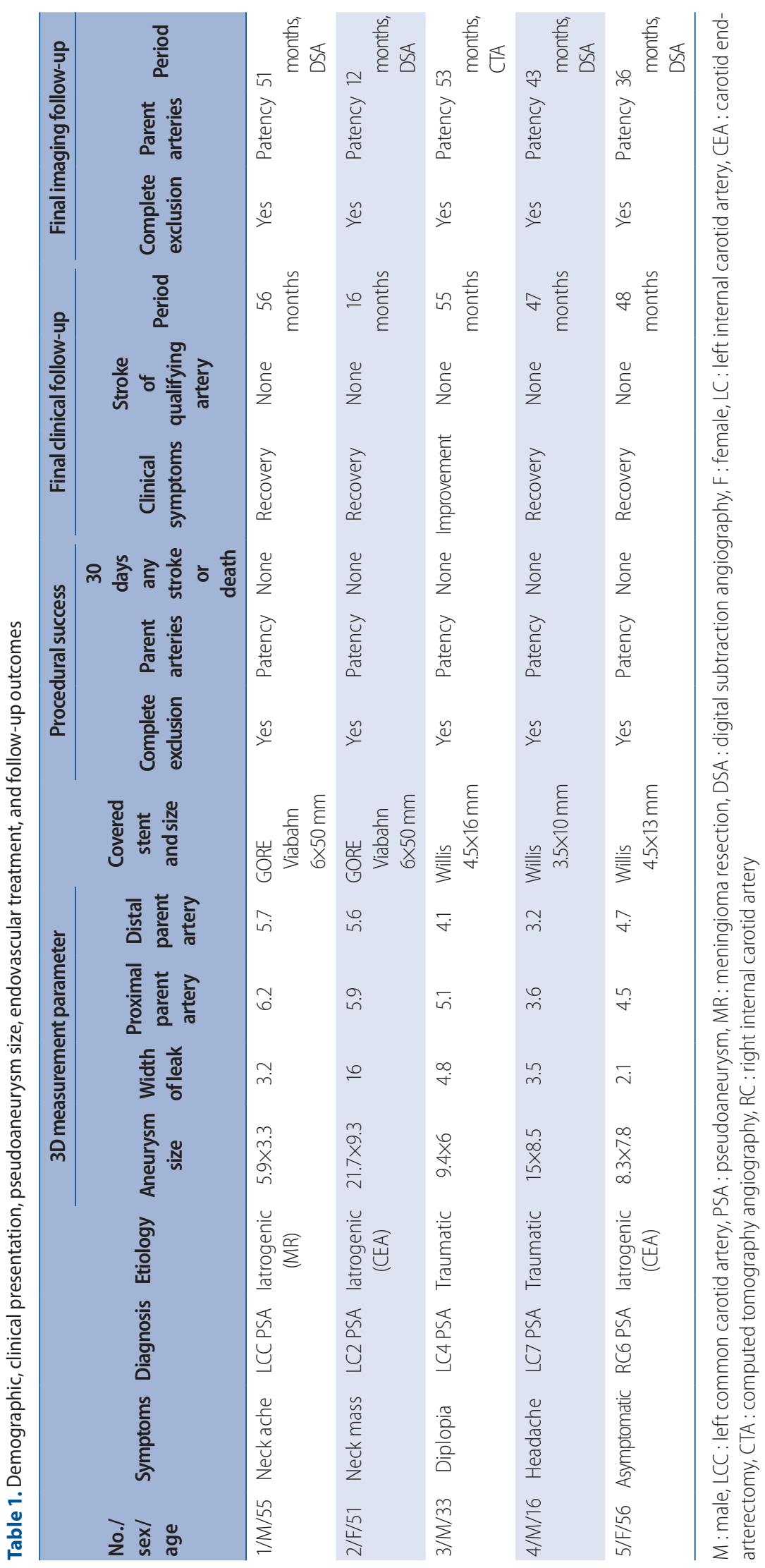



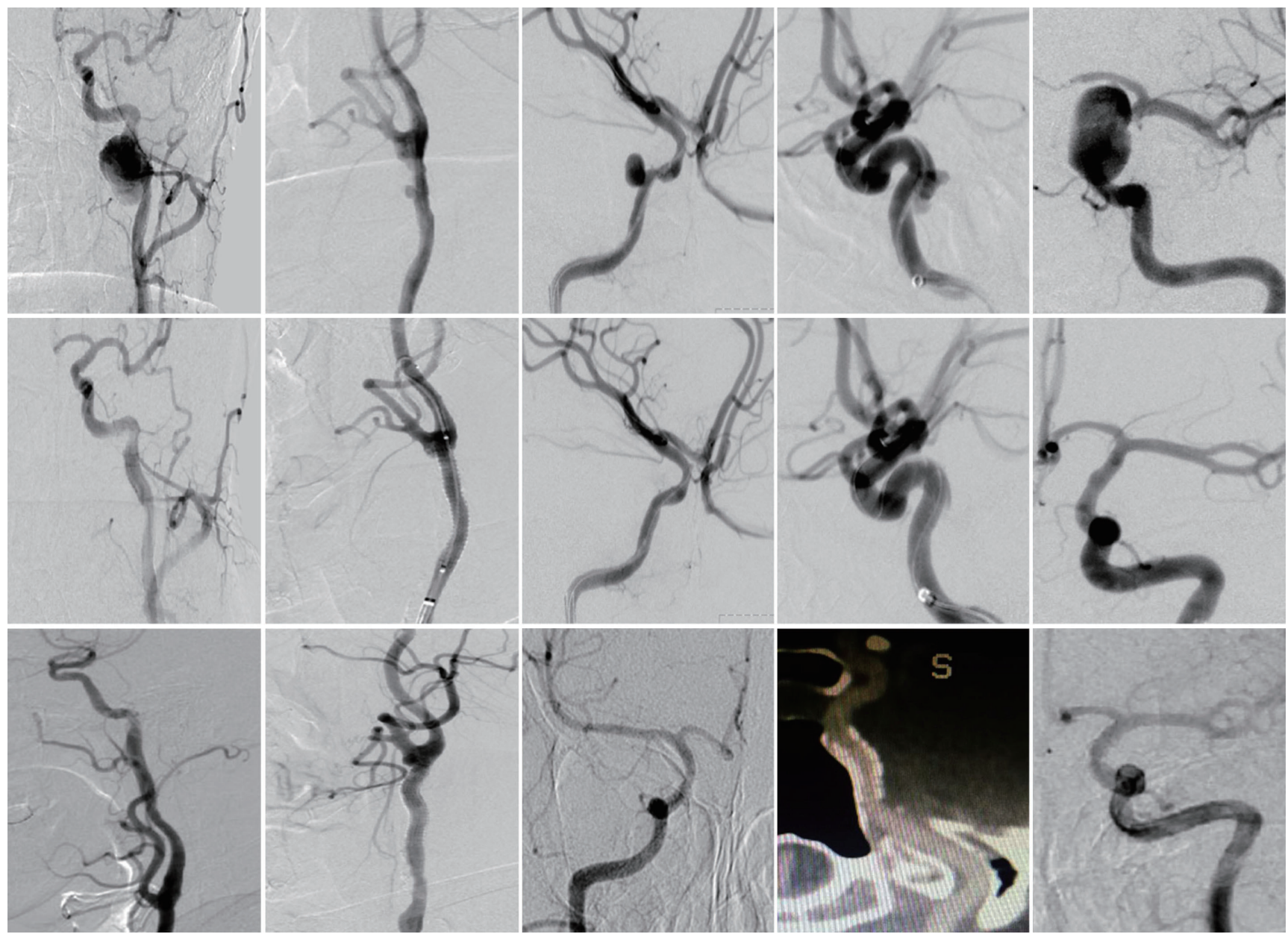

Fig. 2. Preoperative, immediate postoperative and follow-up angiographies of all five included cases.

for four patients and CTA available for one patient), complete exclusion of the pseudoaneurysm and patency of the parent artery were maintained in all patients (Fig. 2). No potential complications (including death, stroke, vessel injury, stent thrombosis, endoleak, and in-stent stenosis) were documented during the follow-up.

\section{DISCUSSION}

Herein, through prospective collection of baseline and longterm follow-up data of five typical cases in a single center, we found that covered stenting was feasible for traumatic carotid pseudoaneurysms, with favorable procedural and long-term outcomes. To our knowledge, this is the first report especially focusing on the long-term efficacy of covered stenting in the treatment of traumatic carotid pseudoaneurysm. Our results present here, together with previously published literature ${ }^{9,13,19,21,23,27)}$, contributed to the recommendations of covered stenting as an effective option for traumatic carotid pseudoaneurysm.

Traumatic carotid pseudoaneurysm (false aneurysm, collection of blood that forms between the tunica media and the tunica adventitia of an artery), as an insidious type of traumatic carotid artery injury, may accidentally occur after blunt/ penetrating trauma or iatrogenic injury (primitive driving force directly disrupted by foreign body puncture or shear stress from blunt trauma) $)^{14}$. Although traumatic carotid pseudoaneurysms are a particularly uncommon clinical condition, with a reported incidence of approximately $1 \%$ in trauma victims, sometimes it not only exposes the wounded to the risk of vascular occlusion or thromboembolism but also leads to rupture with intracranial hemorrhage or cervical compression $^{2}$. Therefore, it is of great significance for clinicians to seek 
better treatment options for traumatic carotid pseudoaneurysm, especially in malignant conditions.

It is well known that conservative treatment is rarely effective for traumatic carotid pseudoaneurysm. Although anticoagulation has been recommended for the treatment of carotid traumatic dissection ${ }^{8)}$, pseudoaneurysms are unlikely to spontaneously resolve after anticoagulation alone. Moreover, anticoagulant therapy is usually a clinical contraindication in cases of pseudoaneurysm complicated with severe injury or multiple trauma. Alternatively, various surgical modalities, such as aneurysm clipping, resection and end-to-end anastomosis, resection and interposition graft, extracranial-to-intracranial bypass, and vessel ligation, have long been the classical methods for the treatment of pseudoaneurysms. However, with reported procedure-related stroke and mortality rates of up to $9 \%{ }^{7)}$ and anatomically difficult situations usually confining the surgical approach, surgical repair is far from favorable for treating traumatic carotid pseudoaneurysm ${ }^{1)}$.

Recently, minimally invasive endovascular therapy has been considered to be a promising treatment strategy and is preferred by experienced interventional radiologists. As with interventional therapy for common aneurysms, endovascular therapy for traumatic pseudoaneurysm can be divided into "destructive principle" (destroying the vessel physiological framework by blocking the parent artery with coils or balloons) and "reconstructive principle" (removal of pseudoaneurysms while preserving the normal vascular anatomy) approaches. In recent years, with the popularity of special intracranial stents, reconstructive strategies using coils and stents have played an increasingly important role in pseudoaneurysms. Despite the lack of randomized controlled trials, the multimodal application of endovascular therapy has shown a lower incidence of death and a breakthrough in surgical anatomical limitations ${ }^{22,23)}$.

Covered stenting is a new reconstructive endovascular strategy worthy of separate discussion. By setting up a direct physical barrier, the covered stent allows the immediate closure of the leakage while maintaining the patency of the parent artery ${ }^{1,11,20)}$. Covered stenting is widely considered to be an ideal choice for the treatment of traumatic carotid pseudoaneurysm because of its exact mechanism and simple operation. The principle of covered stent implantation in endovascular repair for vascular diseases is to implant a biophysical barrier stent in the vasculature. This is by far the most suitable treatment for normal physiology and anatomy. However, because of the scarcity of clinical cases, most of the current experience is based on small-sized case reports in the literature, and the results of long-term follow-up are almost unknown. To further accumulate clinical experience in this field, we carried out the current research and focused on the following issues : the differentiation of intra- and extracranial lesions, related complications, and the persistence of curative effect.

Until now, covered stents have been more widely applied for extracranial pseudoaneurysms; frequently used covered stents include Wallgraft, Jostent, Symbiot and Gore systems ${ }^{15,16}$. Because of the smaller anatomical curvature in extracranial arteries, the delivery and deployment of covered stents tends to be less difficult. The self-expanding Viabahn stent was the only covered stent chosen for extracranial lesions in the present study. The selection of stent size is particularly important for the treatment outcome. To ensure good adherence with the parent artery, the stent diameter was selected mainly according to the larger diameter of the proximal and distal parent arteries. To avoid incomplete exclusion, the length of the stent must be longer than the width of the endoleak. However, for intracranial traumatic pseudoaneurysms, higher requirements need to be met for the delivery flexibility and size of the stent. Although Jostent and Symbiot grafts have been reported for intracranial application, careful patient selection is necessary, and the procedure is technically difficult. The Willis covered stent was designed especially for intracranial use, and it has advantages in flexibility, trackability and stent delivery ${ }^{24,27)}$. In clinical practice, balloon reinflation between named pressure and burst pressure is allowed, which can ensure the covered stent adheres well to the vessel wall. Endoleaks into the pseudoaneurysmal cavity were sometimes still observed after Willis deployment, and the endoleak usually did not persist after reinflation. The long-term follow-up results of the three patients in our study further confirmed that the Willis stent is reliable, which is consistent with a previous study performed by its inventor ${ }^{26,27)}$.

No perioperative or long-term complications (reported as bleeding or rebleeding episodes, endoleak, covered-stent-related iatrogenic dissection, thrombosis, stent fracture, and restenosis) were observed in the present series. This does not mean that the covered stent itself is absolutely safe because relevant complications have been commonly reported in the literature $^{5,10,15,23,27)}$. However, with the deepening of understanding 
of the disease and the accumulation of technical experience, the risk of perioperative complications of pseudoaneurysm stenting tends to be acceptable. According to our practice, the potentially useful strategies include careful evaluation of morphological characteristics, appropriate selection of stent type and size, and adequate anticoagulation therapy during the perioperative period. Preoperative multimodal imaging can provide important information about the location, shape, size and side branches of the pseudoaneurysm, which is helpful for selecting suitable patients for stenting and to predict the treatment effect. The mismatch of stent and lesion is the cause of endoleaks; therefore, it is necessary to select the appropriate stent size, as mentioned earlier. Moreover, standardized perioperative antithrombotic management is another key factor in the prevention of thrombosis complications and restenosis ${ }^{3,17)}$. Dual antiplatelets (aspirin and clopidogrel) should be started at least 3 days before the operation, followed by aspirin alone indefinitely. Generally, with the accumulation of time and cases, issues involving complications have improved.

Our present study focused on the long-term effects of covered stents. In contrast, previous literature data were rare, and the majority of studies focused on immediate or short-term follow-up results. Dimitrios retrospectively analyzed 20 patients in 14 published articles, of which only two cases were followed up for 2 years ${ }^{15)}$. Another relatively new case series focusing on the treatment of traumatic pseudoaneurysms with Willis stents has been completed by Wang et al. ${ }^{27}$. The longest follow-up period was 36 months, but only two cases were followed up for more than 24 months $^{27)}$. As a supplement, our long-term follow-up results further confirmed the persistent curative effect of covered stenting. Moreover, if therapeutic lesions remain stable within 3 years after the stenting operation, the recurrence risk of pseudoaneurysms appears to be very low.

Of note, there is another view that traumatic pseudoaneurysms with localized lesions or without progress might achieve good prognosis through conservative treatment. However, considering the disastrous risk of complications, we prefer an aggressive stenting strategy in our institution. Obviously, the evidence level in the current retrospective research is not sufficient to provide a better answer, which is a limitation of our study. In the future, multicenter case collections with control groups of other therapeutic strategies will be needed.

\section{CONCLUSION}

Satisfactory procedural and long-term outcomes were obtained, suggesting that covered stenting is an effective option for traumatic carotid pseudoaneurysm.

\section{CONFLICTS OF INTEREST}

No potential conflict of interest relevant to this article was reported.

\section{INFORMED CONSENT}

Informed consent was obtained from all individual participants included in this study.

\section{AUTHOR CONTRIBUTIONS}

\author{
Conceptualization : WJJ, KW \\ Data curation : XXP, AFL, YYZ, JL \\ Formal analysis : LX \\ Methodology: WJJ, KW \\ Project administration : WJJ, KW, XXP \\ Visualization : YEL, YYZ \\ Writing - original draft : KW, XXP \\ Writing - review \& editing: WK, WJJ
}

\section{ORCID}

$\begin{array}{ll}\text { Kai Wang } & \text { https://orcid.org/0000-0002-1591-2788 } \\ \text { Xiao-xin Peng } & \text { https://orcid.org/0000-0002-4500-6508 } \\ \text { Ao-fei Liu } & \text { https://orcid.org/0000-0003-2893-1619 } \\ \text { Ying-ying Zhang } & \text { https://orcid.org/0000-0002-7319-6118 } \\ \text { Jin Lv } & \text { https://orcid.org/0000-0002-8429-0687 } \\ \text { Li Xiang } & \text { https://orcid.org/0000-0001-5762-5851 } \\ \text { Yun-e Liu } & \text { https://orcid.org/0000-0003-4813-3630 } \\ \text { Wei-jian Jiang } & \text { https://orcid.org/0000-0003-1156-5720 }\end{array}$




\section{- Acknowledgements}

New Era Stroke Care and Research Institute, The PLA Rocket Force Characteristic Medical Center, is the institution where the research was mainly conducted.

This study is supported by National Research Development Plan (No. 2013CB733800), and the Postdoctoral Science Foundation of China (No. 2017M613374).

\section{References}

1. Ahuja V, Tefera G : Successful covered stent-graft exclusion of carotid artery pseudo-aneurysm: two case reports and review of literature. Ann

Vasc Surg 21 : 367-372, 2007

2. Berne JD, Cook A, Rowe SA, Norwood SH : A multivariate logistic regression analysis of risk factors for blunt cerebrovascular injury. J Vasc Surg 51 : 57-64, 2010

3. Bhatt DL, Kapadia SR, Bajzer CT, Chew DP, Ziada KM, Mukherjee D, et al. : Dual antiplatelet therapy with clopidogrel and aspirin after carotid artery stenting. J Invasive Cardiol 13 : 767-771, 2001

4. Biffl WL, Moore EE, Offner PJ, Brega KE, Franciose RJ, Burch JM : Blunt carotid arterial injuries: implications of a new grading scale. J Trauma $47: 845-853,1999$

5. Choi HC, Park SE, Choi DS, Shin HS, Kim JE, Choi HY, et al. : Ruptured extracranial carotid artery: endovascular treatment with covered stent graft. J Neuroradiol 45 : 217-223, 2018

6. Coldwell DM, Novak Z, Ryu RK, Brega KE, Biffl WL, Offner PJ, et al. : Treatment of posttraumatic internal carotid arterial pseudoaneurysms with endovascular stents. J Trauma $48:$ 470-472, 2000

7. El-Sabrout R, Cooley DA : Extracranial carotid artery aneurysms: Texas Heart Institute experience. J Vasc Surg 31 : 702-712, 2000

8. Esnault P, Cardinale M, Boret H, D'Aranda E, Montcriol A, Bordes J, et al. : Blunt cerebrovascular injuries in severe traumatic brain injury: incidence, risk factors, and evolution. J Neurosurg 127 : 16-22, 2017

9. Fusonie GE, Edwards JD, Reed AB : Covered stent exclusion of blunt traumatic carotid artery pseudoaneurysm: case report and review of the literature. Ann Vasc Surg 18 : 376-379, 2004

10. Hoit DA, Schirmer CM, Malek AM : Stent graft treatment of cerebrovascular wall defects: intermediate-term clinical and angiographic results. Neurosurgery 62(5 Suppl 2) : ONS380-ONS388; discussion ONS388-ONS389, 2008

11. Jamshidi P, Mahmoody K, Erne P : Covered stents: a review. Int J Cardiol $130: 310-318,2008$

12. Klein GE, Szolar DH, Raith J, Fruhwirth H, Pascher O, Hausegger KA : Posttraumatic extracranial aneurysm of the internal carotid artery: combined endovascular treatment with coils and stents. AJNR Am J Neurora- diol $18:$ 1261-1264, 1997

13. Kubaska SM 3rd, Greenberg RK, Clair D, Barber G, Srivastava SD, Green RM, et al. : Internal carotid artery pseudoaneurysms: treatment with the Wallgraft endoprosthesis. J Endovasc Ther 10 : 182-189, 2003

14. Magge D, Farber A, Vladimir F, Woodson J, Collins K, Shaw P, et al. : Diagnosis and management of traumatic pseudoaneurysm of the carotid artery: case report and review of the literature. Vascular $16: 350-355$, 2008

15. Maras D, Lioupis C, Magoufis G, Tsamopoulos N, Moulakakis K, Andrikopoulos $V$ : Covered stent-graft treatment of traumatic internal carotid artery pseudoaneurysms: a review. Cardiovasc Intervent Radiol 29 : 958-968, 2006

16. Maskanakis A, Patelis N, Moris D, Tsilimigras DI, Schizas D, Diakomi M, et al. : Stenting of subclavian artery true and false aneurysms: a systematic review. Ann Vasc Surg 47 : 291-304, 2018

17. McCready RA, Divelbiss JL, Bryant MA, Denardo AJ, Scott JA : Endoluminal repair of carotid artery pseudoaneurysms: a word of caution. J Vasc Surg 40 : 1020-1023, 2004

18. McNeil JD, Chiou AC, Gunlock MG, Grayson DE, Soares G, Hagino RT : Successful endovascular therapy of a penetrating zone III internal carotid injury. J Vasc Surg 36 : 187-190, 2002

19. Pai BS, Limaye $U$, Varma RG : Endovascular management of a refractory traumatic aneurysm of the internal carotid artery using a covered stent graft. Neurol India 56 : 488-500, 2008

20. Pierot L, Gawlitza M, Soize S : Unruptured intracranial aneurysms: management strategy and current endovascular treatment options. Expert Rev Neurother 17 : 977-986, 2017

21. Redekop G, Marotta T, Weill A : Treatment of traumatic aneurysms and arteriovenous fistulas of the skull base by using endovascular stents. J Neurosurg 95 : 412-419, 2001

22. Seth $\mathrm{R}$, Obuchowski AM, Zoarski GH : Endovascular repair of traumatic cervical internal carotid artery injuries: a safe and effective treatment option. AJNR Am J Neuroradiol 34 : 1219-1226, 2013

23. Spanos K, Karathanos C, Stamoulis K, Giannoukas AD : Endovascular treatment of traumatic internal carotid artery pseudoaneurysm. Injury $47:$ 307-312, 2016

24. Tang C, Qi S : Efficacy and safety of Willis covered stent for treatment of internal carotid artery aneurysms. J Craniofac Surg 28 : e263-e265, 2017

25. Tsai $\mathrm{YH}$, Wong HF, Weng HH, Chen YL : Stent-graft treatment of traumatic carotid artery dissecting pseudoaneurysm. Neuroradiology 52 : 1011-1016, 2010

26. Wang JB, Li MH, Fang C, Wang W, Cheng YS, Zhang PL, et al. : Endovascular treatment of giant intracranial aneurysms with Willis covered stents: technical case report. Neurosurgery 62 : E1176-E1177; discussion E1177, 2008

27. Wang W, Li MH, Li YD, Gu BX, Wang J, Zhang PL, et al. : Treatment of traumatic internal carotid artery pseudoaneurysms with the Willis covered stent: a prospective study. J Trauma 70 : 816-822, 2011 